both in ex vivo and in the Th17-polarizing cultures. While the frequencies of Treg $\left(\mathrm{CD} 4^{+} \mathrm{CD} 25^{+} \mathrm{CD} 127^{\mathrm{dim} /-}\right)$ cells were lower in the corresponding populations. Higher levels of IL17 and IL6 were detected in plasma of SLE patients. Responding to CD95-induced apoptosis the frequency of $\mathrm{CD} 4^{+} \mathrm{IL} 17^{+}$cells from SLE patients was substantially lower, but that of $\mathrm{CD} 4^{+} \mathrm{CD} 25^{+} \mathrm{CD} 127^{\mathrm{dim} /-}$ cells from Treg-polarizing cultures was considerably higher. With treatment of atorvastatin, $\mathrm{CD} 4^{+} \mathrm{IL} 17^{+}$ cell population in cultures derived from SLE patients showed an increased susceptibility to CD95-induced apoptosis. However, the CD4 ${ }^{+} \mathrm{CD} 25^{+} \mathrm{CD} 127^{\mathrm{dim} /-}$ cell population had reduced response to apoptosis. Accordingly, the ratio of transcription factor RORC/FoxP3 decreased in T cell cultures of SLE patients. Conclusions: Th17 cells were more resistant than Treg cells to CD95-induced apoptosis in SLE as compared to control subjects. Statins counteracted the dysregulated susceptibility of SLE T cells to apoptosis. Our findings reveal a novel mechanism underlying the imbalance of Th17/Treg and show a potential interest to the treatment of the patients with SLE.

Disclosure of Interest: None declared

DOI: 10.1136/annrheumdis-2017-eular.6193

\section{THU0227 GENOME-WIDE ASSOCIATION META-ANALYSIS IDENTIFIES FIVE NEW LOCI FOR SYSTEMIC LUPUS ERYTHEMATOSUS}

A. Julià ${ }^{1}$, P. Carreira ${ }^{2}$, R. Blanco ${ }^{3}$, F.J. López-Longo ${ }^{4}$, J.J. Pérez-Venegas ${ }^{5}$, A. Olivé $^{6}$, J.L. Andreu ${ }^{7}$, M.A. Aguirre-Zamorano ${ }^{8}$, P. Vela ${ }^{9}$, J.M. Nolla ${ }^{10}$, J.L. Marenco de la Fuente ${ }^{11}$, A. Zea ${ }^{12}$, J.M. Pego ${ }^{13}$, M. Freire ${ }^{14}$, E. Díez ${ }^{15}$, M. López-Lasanta ${ }^{1}$, M. López-Corbeto ${ }^{1}$, N. Palau ${ }^{1}$, R. Tortosa ${ }^{1}$, E. Trallero ${ }^{1}$, A. Aterido ${ }^{1}$, D. Absher ${ }^{16}$, R.M. Myers ${ }^{17}$, A. Fernandez-Nebro ${ }^{18}$, S. Marsal ${ }^{1}$ ${ }^{1}$ Grup de Recerca de Reumatologia, Vall Hebron Research Institute, Barcelona;

${ }^{2}$ Rheumatology Department, Hospital Universitario 12 de Octubre, Madrid; ${ }^{3}$ Rheumatology Department, Hospital Universitario Marqués de Valdecilla, Santander: ${ }^{4}$ Rheumatology Department, Hospital Universitario Gregorio Marañón, Madrid; ${ }^{5}$ Rheumatology Department, Hospital del SAS de Jerez de la Frontera, Cádiz; ${ }^{6}$ Rheumatology Department, Hospital Universitari Germans Trias i Pujol, Badalona; ${ }^{7}$ Rheumatology Department, Hospital Universitario Puerta de Hierro, Madrid; ${ }^{8}$ Rheumatology Department, Hospital Universitario Reina Sofía, Córdoba: ${ }^{9}$ Rheumatology Department, Hospital General Universitario de Alicante, Alicante; ${ }^{10}$ Rheumatology Department, Hospital Universitari de Bellvitge, Barcelona; ${ }^{11}$ Rheumatology Department, Hospital de Valme, Sevilla; ${ }^{12}$ Rheumatology Department, Hospital Universitario Ramón y Cajal, Madrid: ${ }^{13}$ Rheumatology Department, Hospital do Meixoeiro, Vigo; ${ }^{14}$ Rheumatology Department, Hospital Universitario A Coruña, A Coruña; ${ }^{15}$ Rheumatology Department, Hospital Complejo Asistencial Universitario de León, León, Spain: ${ }^{16}$ Absher Lab: ${ }^{17}$ Myers Lab, HudsonAlpha Institute for Biotechnology, Huntsville, United States; ${ }^{18}$ Rheumatology Department, Hospital Regional Universitario de Málaga, Málaga, Spain

Background: Recent genome-wide association studies (GWAS) have identified more than 50 loci associated with systemic lupus erythematosus but they explain less than $30 \%$ of the heritability of the disease. Meta-analysis including new populations can contribute to identify additional genetic risk factors.

Objectives: The aim of the presents study was to identify additional genetic risk loci for SLE.

Methods: We performed a meta-analysis using data from a recent large scale GWAS from 4,036 cases and 6,959 controls from Caucasian European ancestry [1] and a newly genotyped cohort of 907 SLE patients and 1,524healthy controls from Spain. Genetic association was tested at the single-marker level using linear regression and at the pathway-level using Fisher's modified method.

Results: Combining the two cohorts we identified genome-wide significant association $(\mathrm{P}<5 \mathrm{E}-8)$ at five new loci: three SNPs at intragenic regions and two intergenic loci at chromosomes $7 q 11.23$ (Manhattan plot for chromosome 7 is shown in Figure; new SLE risk region SNPs highlighted in green) and 17q21.31.

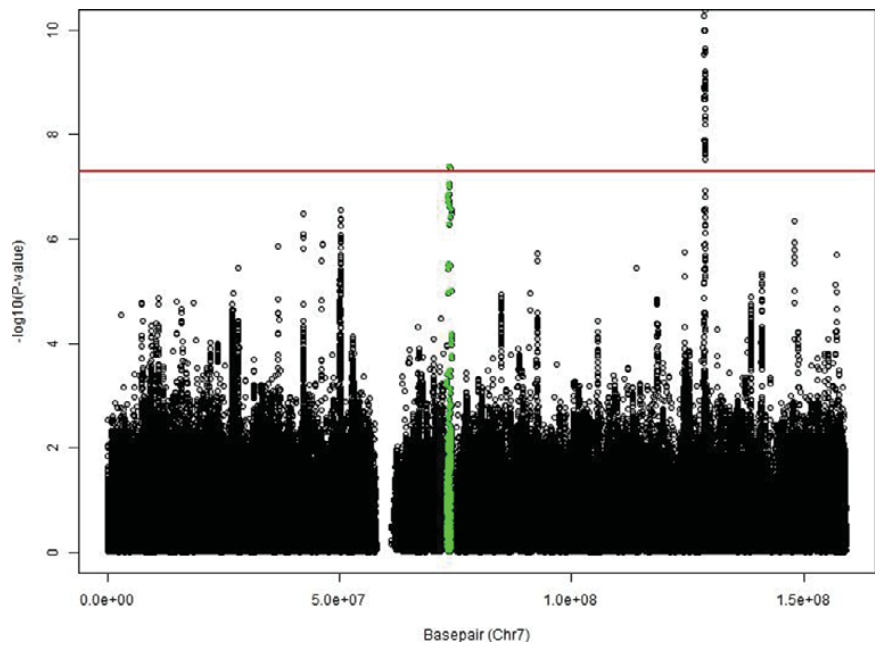

Several of the new associated genes are functionally associated with B cell regulation. After multiple test correction, $B$ Cell Receptor signaling, Biopeptides and Cell surface interactions at the vascular wall pathways were also significantly associated with SLE risk.

Conclusions: In conclusion, we have identified five new risk loci for SLE through a meta-analysis including a new GWAS.

\section{References:}

[1] Bentham J, Morris DL et al. Nature Genetics 2015

Acknowledgements: We would like to thank the clinical researchers and patients participating in the IMID Consortium for their collaboration. We would also like to thank the international SLE consortium for access to the data.

Disclosure of Interest: None declared

DOI: 10.1136/annrheumdis-2017-eular.2854

\section{THU0228 INTRA-RENAL ACTIVATION OF ADAPTIVE IMMUNE EFFECTORS IS ASSOCIATED WITH HIGHER DISEASE SEVERITY IN LUPUS NEPHRITIS}

C. Pamfil ${ }^{1}$, N. Demoulin ${ }^{2}$, M. Jadoul ${ }^{2}$, S. Aydin ${ }^{3}$, F.A. Houssiau ${ }^{1,4}$, B. Lauwerys $\mathrm{s}^{1,4}{ }^{1}$ Pôle de pathologies rhumatismales systémiques et inflammatoires, Institut de Recherche Expérimentale et Clinique, Université catholique de Louvain; ${ }^{2}$ Department of Nephrology; ${ }^{3}$ Department of Pathology; ${ }^{4}$ Department of Rheumatology, Cliniques universitaires Saint-Luc, Brussels, Belgium

Background: Chronic renal impairment remains a feared complication of lupus nephritis (LN). Yet, little is known about mechanisms and markers of disease severity in the lupus kidney.

Methods: We performed high-throughput transcriptomic studies (Illumina HumanHT12 v4 Expression Beadchips) on archived (- 80 degrees) kidney biopsies from 32 SLE patients and 8 controls (pre-transplant donors). Unsupervized clustering and differential gene expression studies were performed using GeneSpring software. Pathway analyses were carried on using DAVID and GSEA. Clinical and biological data were retrieved from the patients' medical records. Immunohistochemistry experiments (CD3, CD20, CD21) were performed on the same samples, and on an additional cohort of 37 SLE kidney biopsies. Syndecan-1 (SDC1) was used as a marker of renal tubular cell response to stress.

Results: Compared to controls, LN samples overexpressed transcripts involved in interferon signature, apoptosis, chemokines, antigen presentation, T and B cell activation

Unsupervized clustering studies isolated 14 SLE samples based on their gene expression features. These samples were characterized by a significantly lower estimated GFR at the time of biopsy (T0) (50.7 versus $\left.97.4 \mathrm{ml} / \mathrm{min} / 1.72 \mathrm{~m}^{2}\right)$, but also at follow-up ( 49.1 versus $85.8 \mathrm{ml} / \mathrm{min} / 1.72 \mathrm{~m}^{2}$ ) compared to the other $\mathrm{SLE}$ samples. Yet, apparent renal disease duration at T0, disease duration at last follow-up (median 91.5 versus 86 months), double-stranded DNA antibody titers at T0 and other relevant characteristics (histological scores, proteinuria, numbers of subsequent flares) were not different between both groups.

From a transcriptomic point of view, these 14 samples were characterized by the overexpression of transcripts and pathways involved in adaptive immune responses: antigen presentation, $\mathrm{T}$ cell differentiation and $\mathrm{B}$ cell activation. Immunohistochemistry studies confirmed a significant association between the presence of CD3 and CD20 positive cells in the interstitial space and lower estimated GFR at baseline in the same, but also in an independent set of samples. The presence of CD3 and CD20 positive cells was also associated with lower SDC1 expression on renal tubular cells. Low SDC1 expression on renal tubular cells was strongly associated with impaired kidney function at baseline. Conclusions: LN kidney biopsy samples from patients with lower estimated GFR are characterized by the overexpression of transcripts pointing to the activation of a local antigen-dependent immune response. Activation of "second wave" immune effectors in the LN kidney is a known feature of the disease, and impacts kidney function through alterations in the function of renal tubular cells.

Acknowledgements: The research leading to these results has received support from the Innovative Medicines Initiative Joint Undertaking under grant agreement $n$ 115565, resources of which are composed of financial contribution from the European Union's Seventh Framework Programme (FP7/2007-2013) and EFPIA companies' in kind contribution

Disclosure of Interest: None declared

DOI: 10.1136/annrheumdis-2017-eular.3427 